\title{
Semi-inclusive DIS: Factorization
}

\author{
Feng $\operatorname{Yuan}^{1,2} *$ \\ 1- Nuclear Science Division, Lawrence Berkeley National Laboratory, Berkeley, CA 94720, USA \\ 2- RIKEN BNL Research Center, Brookhaven National Laboratory, Upton, NY 11973, USA
}

In this talk, we will present a QCD factorization theorem for the semi-inclusive deepinelastic scattering with hadrons in the current fragmentation region detected at low transverse momentum.

There has been considerable experimental and theoretical interest in semi-inclusive hadron production in deep inelastic scattering (SIDIS) processes. For example, by studying the polarized and unpolarized SIDIS, one will be able to identify the sea quark distribution and polarization in nucleon, and the experimental results from the HERMES collaboration have revealed nontrivial sea structure in nucleon [1]. More recently, SIDIS opened a new window to study the transverse momentum dependent (TMD) parton distributions and fragmentation functions from the low transverse momentum hadron production. The transverse momentum distribution of the final state hadron is directly related to the transverse momentum dependence of the parton distributions and fragmentation. These studies will provide new opportunities to explore the partonic structure of nucleon, especially the threedimension distribution of partons inside nucleon. The DIS experiments, including HERMES, COMPAS, and JLab Hall B collaborations, have studied various azimuthal asymmetries in SIDIS. In particular, the HERMES collaboration found sizable single spin asymmetries in these processes involving nontrivial QCD effects and hadron structure.

On the theory side, there has been great progress in the last few years too. In this short talk, it is impossible to cover all these important physics. Rather, I would like to focus on one of the questions concerning applying the QCD to the description of the semi-inclusive DIS, in particular, the QCD factorization for SIDIS. Unlike the inclusive DIS process, SIDIS is more involved because of additional hadron measurement in the final state. We can classify the SIDIS processes into three different categories. First, if we integrate out the transverse momentum of the final state hadron, these SIDIS will be similar to the inclusive DIS, and a collinear factorization is applicable. The cross section can be written as a convolution of the integrated parton distribution and fragmentation function and the hard partonic cross section which can be calculated from perturbative QCD. Second class is the large transverse momentum SIDIS. When transverse momentum is large enough (in order of $Q$, the virtuality of the virtual photon), again a collinear factorization shall be used to analyze the cross section. The third class is the low transverse momentum hadron production, where a collinear factorization approach may not be applicable because the transverse momentum of the final state hadron is small compared to the hard scale $Q$. In order to describe this class of processes, we have to introduce a new factorization theorem, involving the transverse momentum dependent parton distribution and fragmentation functions. Rigorous theoretical studies in this direction started from the classical work on semi-inclusive processes in $e^{+} e^{-}$annihilation by Collins and Soper [2], where a QCD factorization was proved, and

*This work was supported in part by the U.S. Department of Energy under contract DE-AC0205CH11231. We are grateful to RIKEN, Brookhaven National Laboratory and the U.S. Department of Energy (contract number DE-AC02-98CH10886) for providing the facilities essential for the completion of this work.

DIS 2008 
non-perturbative transverse-momentum-dependent (TMD) parton distributions and fragmentation functions were introduced $[2,3]$. In the past few years, gauge properties of the TMD parton distributions have been investigated $[4,5,6]$. More recently, the factorization theorems for the semi-inclusive deep inelastic scattering (SIDIS) and Drell-Yan processes have been re-examined in the context of the gauge-invariant definitions $[7,8]$.

The main result of [7] is a QCD factorization argument for the SIDIS cross section at low transverse momentum, accurate up to the power corrections $\left(P_{h \perp}^{2} / Q^{2}\right)^{n}$ and to all orders in perturbation theory. For example, the leading spin-independent structure function for SIDIS can be factorized as follows,

$$
\begin{aligned}
F\left(x_{B}, z_{h}, P_{h \perp}, Q^{2}\right)= & \sum_{q=u, d, s, \ldots} e_{q}^{2} \int d^{2} \vec{k}_{\perp} d^{2} \vec{p}_{\perp} d^{2} \vec{\ell}_{\perp} \\
& \times q\left(x_{B}, k_{\perp}, \mu^{2}, x_{B} \zeta, \rho\right) \hat{q}_{T}\left(z_{h}, p_{\perp}, \mu^{2}, \hat{\zeta} / z_{h}, \rho\right) S\left(\vec{\ell}_{\perp}, \mu^{2}, \rho\right) \\
& \times H\left(Q^{2}, \mu^{2}, \rho\right) \delta^{2}\left(z_{h} \vec{k}_{\perp}+\vec{p}_{\perp}+\vec{\ell}_{\perp}-\vec{P}_{h \perp}\right)
\end{aligned}
$$

where $\mu$ is a renormalization (and collinear factorization) scale; $\rho$ is a gluon rapidity cut-off parameter; the $\mu$ and $\rho$ dependence cancels among various factors. In a special system of coordinates in which $x_{B} \zeta=\hat{\zeta} / z_{h}$, one has $\zeta^{2} x_{B}^{2}=\hat{\zeta}^{2} / z_{h}^{2}=Q^{2} \rho$. The physical interpretation of the factors are as follows: $q$ is TMD quark distribution function; $\hat{q}$ is the TMD quark fragmentation function depending on; $H$ represents the contribution of parton hard scattering and is a perturbation series in $\alpha_{s}$; and, finally, the soft factor $S$ comes from soft gluon radiations and is defined by a matrix element of Wilson lines in QCD vacuum.

We emphasize that the above factorization formula is valid in the limit of $Q^{2} \rightarrow \infty$, and all higher order corrections in terms of $P_{h \perp} / Q$ have been neglected. In the factorization formula, the transverse momentum dependent parton distribution plays the central role. Let us discuss these distributions first. Consider a hadron, a nucleon for example, with four-momentum $P$. Let $\left(x P^{+}, \vec{k}_{\perp}\right)$ represent the momentum of a parton (quark or gluon) in the hadron. In a non-singular gauge (e.g. Feynman gauge), the TMD parton distributions can be defined through the following matrix $[2,5]$,

$$
\begin{aligned}
\mathcal{M}^{ \pm}\left(x, k_{\perp}, \mu, x \zeta, \rho\right)= & p^{+} \int \frac{d \xi^{-}}{2 \pi} e^{-i x \xi^{-} P^{+}} \int \frac{d^{2} \vec{b}_{\perp}}{(2 \pi)^{2}} e^{i \vec{b}_{\perp} \cdot \vec{k}_{\perp}} \\
& \times\left\langle P S\left|\bar{\psi}_{q}\left(\xi^{-}, \vec{b}_{\perp}\right) \mathcal{L}_{v}^{\dagger}\left( \pm \infty ; \xi^{-}, \vec{b}_{\perp}\right) \mathcal{L}_{v}( \pm \infty ; 0) \psi_{q}(0)\right| P S\right\rangle .
\end{aligned}
$$

The $+(-)$ superscript is appropriate for DIS (Drell-Yan) process $[5,6] . v^{\mu}$ is a time-like dimensionless $\left(v^{2}>0\right)$ four-vector with zero transverse components $\left(v^{-}, v^{+}, \overrightarrow{0}\right)$ and $v^{-} \gg$ $v^{+}$. Thus the $v^{\mu}$ is a quasi light-cone vector, approaching $n^{\mu}$. The variable $\zeta^{2}$ denotes the combination $(2 P \cdot v)^{2} / v^{2}=\zeta^{2} \cdot \mathcal{L}_{v}$ is a gauge link along $v^{\mu}$,

$$
\mathcal{L}_{v}( \pm \infty ; \xi)=\exp \left(-i g \int_{0}^{ \pm \infty} d \lambda v \cdot A(\lambda v+\xi)\right)
$$

Here the non-light-like gauge link is introduced to regulate the light-cone singularities. The TMD parton distribution is defined as such to absorb the collinear divergence in the partonic processes. This has been checked by an explicit calculation at one-loop order [7], where the soft divergence associated with soft gluons in the TMDs has been cancelled out in the total result, and we are left with only the collinear singularity. 
It is instructive to demonstrate this factorization at one-loop order. For example, the one-loop real corrections to the structure function $F$ are shown in Fig. 1. There is no contribution to the hard scattering kernel from any of these diagrams. In Fig. 1a, the gluon radiation generates a transverse-

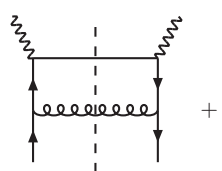

(a)

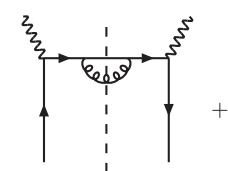

(b)

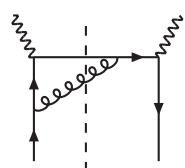

(c)

Figure 1: One-loop real diagrams for SIDIS. momentum for the struck quark. There is no contribution from the fragmentation function because the contribution from the final state with a gluon parallel to final state quark is power suppressed. Therefore, the diagram must be factorizable into the parton distribution. Similarly for Fig. 1b, which again can be reproduced by the factorization formula with the one-loop fragmentation function and the soft factor S, and the tree-level parton distribution and the hard part. For Fig. 1c and its hermitian conjugate, we find three distinct contributions: where the first term corresponds to a gluon collinear to the initial quark, the second term a gluon collinear to the final state quark, and the third term a soft gluon. All these terms are reproduced by the factorization formula with one-loop parton distribution, fragmentation function, and the soft factor. The virtual contribution can be analyzed accordingly [7].

For arguments toward a factorization to all orders, we follow the discussions in $[2,7]$. The procedure for this argument is the following. First, for any high order Feynman diagrams, using the power counting rules identifies the leading region contributions [9]. The leading regions clearly separate the soft, collinear, and hard gluons' contributions to the cross section (the cut diagram), where the soft gluons are only attached to the jet functions (parton distributions and/or fragmentation functions); hard gluons are included in the hard part; collinear gluons attached the jet functions to the hard part. On top of that, we can further use the Grammer-Yennie approximation to factorize out the soft factor, which can be expressed as matrix element of Wilson lines $[2,7]$. The Ward Identity will be used to further factorize the collinear gluons from the hard part, which results in a Wilson line (gauge link) association in the definition of the jet functions. The variation of the gauge link gives the Collins-Soper evolution equation for the jet functions [2]. After these procedures, the hard part only contains hard gluons, which can be calculated from perturbative QCD.

The above factorization argument is also applicable to the spin and azimuthal dependent SIDIS [7] at leading order of $1 / Q$. In particular, the Sivers-type single transverse spin asymmetry in SIDIS has been studied in [10], where the gluon radiation generates large transverse momentum Sivers function and fragmentation. When combining both contributions with the soft factor, the Sivers-type SSA in SIDIS can be factorized into the similar factorization formula as the above. The calculations of [10] are based one collinear factorization approach, and the SSA comes from the twist-three quark-gluon correlation function as we show in the diagram of Fig. 2(a). At large transverse momentum $P_{h \perp} \sim Q$, the SSA in of higher-twist, and will be suppressed by $1 / P_{h \perp}$. At small $P_{h \perp} \ll Q$, a factorization in terms of TMD parton distribution applies [7], involving in case of the SSA the Sivers functions. If $P_{h \perp}$ is much larger than $\Lambda_{\mathrm{QCD}}$, the dependence of these functions on transverse momentum can be computed using QCD perturbation theory. At the same time, the result obtained from the diagram Fig. 2(a) can also be extrapolated into the regime $\Lambda_{\mathrm{QCD}} \ll P_{h \perp} \ll Q$, and it can be shown that the result of this extrapolation is identical to that obtained using the TMD approach [10]. In this sense, the two mechanisms widely held responsible for the observed SSAs are unified. 
Again, the factorization arguments we used in the analysis for the spin-average cross section is crucial to obtain this result. Especially, according to the radiation gluon's momentum in Fig. 2(a), the contribution can be factorized into the different factors in the factorization for-

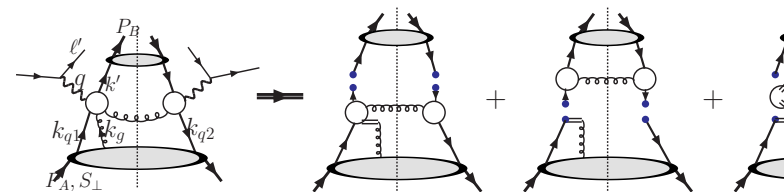

(a) (b)

Figure 2: The factorization arguments for the consistency between the two mechanisms: left is a generic Feynman diagram in the twist-three quark-gluon correlation formalism; and the right is the corresponding TMD factorization form decomposed into different regions, (a) the Sivers function, (b) the fragmentaion function, and (c) the soft factor.

mula. For example, if the

radiation gluon is parallel to the polarized proton, the contribution can be factorized into the spin-dependent Sivers function as we show in Fig. 2(b); if it is parallel to the final state hadron, the contribution can be factorized into the unpolarized quark fragmentation function (Fig. 2(c)); if it is soft, the contribution will belong to the soft factor (Fig. 2(d)). This demonstrated that the factorization argument is very powerful tool to study these physics.

However, the above argument does not work for the azimuthal dependent cross section terms at sub-leading order in $P_{h \perp} / Q$, where a TMD factorization approach seems not valid, at least in the present form [11]. More researches are needed along this line.

\section{References}

[1] H. Jackson, for the HERMES Collaboration, these proceedings.

[2] J. C. Collins and D. E. Soper, Nucl. Phys. B 193, 381 (1981) [Erratum-ibid. B 213, 545 (1983)]; Nucl. Phys. B 197, 446 (1982).

[3] J. C. Collins and D. E. Soper, Nucl. Phys. B 194, 445 (1982).

[4] S. J. Brodsky, D. S. Hwang and I. Schmidt, Phys. Lett. B 530, 99 (2002).

[5] J. C. Collins, Phys. Lett. B 536, 43 (2002); Acta Phys. Polon. B 34, 3103 (2003).

[6] X. Ji and F. Yuan, Phys. Lett. B 543, 66 (2002); A. V. Belitsky, X. Ji and F. Yuan, Nucl. Phys. B 656, 165 (2003).

[7] X. Ji, J. P. Ma and F. Yuan, Phys. Rev. D 71, 034005 (2005); Phys. Lett. B 597, 299 (2004); JHEP 0507, 020 (2005).

[8] J. C. Collins and A. Metz, Phys. Rev. Lett. 93, 252001 (2004).

[9] J. C. Collins, D. E. Soper and G. Sterman, Adv. Ser. Direct. High Energy Phys. 5, 1 (1988); in Perturbative QCD (A.H. Mueller, ed.) (World Scientific Publ., 1989).

[10] X. Ji, J. W. Qiu, W. Vogelsang and F. Yuan, Phys. Rev. Lett. 97, 082002 (2006); Phys. Rev. D 73 , 094017 (2006); Phys. Lett. B 638, 178 (2006).

[11] A. Bacchatta, these proceedings. 